\title{
Basic helix-loop-helix transcription factors DEC1 and DEC2 regulate the paclitaxel-induced apoptotic pathway of MCF-7 human breast cancer cells
}

\author{
YUNYAN WU ${ }^{1,2}$, FUYUKI SATO ${ }^{1}$, UJJAL KUMAR BHAWAL ${ }^{3}$, TAKESHI KAWAMOTO ${ }^{4}$, KATSUMI FUJIMOTO ${ }^{4}$, \\ MITSUHIDE NOSHIRO ${ }^{4}$, SATOKO MOROHASHI ${ }^{1}$, YUKIO KATO ${ }^{4}$ and HIROSHI KIJIMA ${ }^{1}$ \\ ${ }^{1}$ Department of Pathology and Bioscience, Hirosaki University Graduate School of Medicine, Hirosaki 036-8562, Japan; \\ ${ }^{2}$ Department of Pathology, College of Basic Medical Sciences, China Medical University, Shenyang 110001, P.R. China; \\ ${ }^{3}$ Department of Oral and Maxillofacial Surgery and High-Tech Research Center, Kanagawa Dental College, \\ Yokosuka 238-8580; ${ }^{4}$ Department of Dental and Medical Biochemistry, Hiroshima University \\ Graduate School of Biomedical Science, Hiroshima 734-8553, Japan
}

Received November 17, 2010; Accepted January 10, 2011

DOI: $10.3892 / \mathrm{ijmm} .2011 .617$

\begin{abstract}
Differentiated embryonic chondrocyte gene (DEC) 1 (BHLHE40/Stra13/Sharp2) and DEC2 (BHLHE41/ Sharp1) are basic helix-loop-helix (bHLH) transcription factors that are associated with the regulation of apoptosis, cell proliferation and circadian rhythms, as well as malignancy in various cancers. However, the roles of DEC1 and DEC2 expression in breast cancer are poorly understood. In this study, we sought to examine the roles of DEC1 and DEC2 in MCF-7 human breast cancer cells that had been treated with paclitaxel. The expression of DEC1 and DEC2 was up-regulated in paclitaxel-treated MCF-7 cells. Knockdown of DEC 1 by siRNA decreased the amount of cleaved poly (ADP-ribose) polymerase (PARP), after treatment with paclitaxel, whereas DEC2 knockdown increased the amount of cleaved PARP in both the presence and absence of paclitaxel. Immunofluorescent staining revealed that paclitaxel treatment increased the amount of DEC1 in the nucleus, and increased the amount of DEC2 in both the nucleus and cytoplasm. These results indicate that DEC1 has pro-apoptotic effects, whereas DEC2 has anti-apoptotic effects on the paclitaxel-induced apoptosis in human breast cancer MCF-7 cells.
\end{abstract}

Correspondence to: Dr Fuyuki Sato, Department of Pathology and Bioscience, Hirosaki University Graduate School of Medicine, 5 Zaifu-cho, Hirosaki 036-8562, Japan

E-mail: fsato@cc.hirosaki-u.ac.jp

Abbreviations: DEC1, differentiated embryonic chondrocyte gene 1; DEC2, differentiated embryonic chondrocyte gene 2; PARP, poly (ADP-ribose) polymerase

Key words: differentiated embryonic chondrocyte gene 1, differentiated embryonic chondrocyte gene 2, paclitaxel, MCF-7

\section{Introduction}

Paclitaxel is an anti-tumor drug that is used against a wide variety of solid tumors $(1,2)$ and affects the expression of Bcl-2, Bcl-xL and phosphorylated-c-Jun $\mathrm{NH}_{2}$-terminal kinase (JNK), and the activation of caspases and poly (ADP-ribose) polymerase PARP (3-6), resulting in the induction of apoptosis by p53-dependent or -independent mechanisms (7-9). Paclitaxel also causes growth arrest at the G2/M phase of the cell cycle $(5,10)$. Resistance to paclitaxel treatment often occurs in breast cancer, but the detailed mechanisms by which paclitaxel resistance is conferred remain undetermined.

Differentiated embryonic chondrocyte gene (DEC) 1 and DEC2 have been reported to be involved in the induction of apoptosis, the regulation of cell proliferation, and circadian rhythms, progression to malignancy, and the response to hypoxia (11-13). DEC1 is highly expressed in tumor tissues of breast and colon cancers compared to their non-tumor regions $(14,15)$, although the role of DEC2 in tumors is poorly understood. Overexpression and knockdown of DEC1 and DEC2, respectively, affected the expression of apoptosis related-factors such as Bcl-2, Fas, Bax, c-Myc, survivin, and vascular endothelial growth factor (VEGF) as well as the amounts of cleaved PARP and cleaved caspases $(11,12,15,16)$. DEC1 and DEC2 have been shown to regulate the transcription of target genes in an E-box-dependent or -independent manner (17-19). DEC1 has been reported to induce apoptosis in mouse fibroblast NIH3T3 cells, while DEC1 has been found to inhibit apoptosis under serum deprivation in human embryonic kidney HEK293 cells (16,20). DEC1 is also involved in the regulation of apoptosis by transforming growth factor (TGF- $\beta$ ) treatment in mouse mammary carcinoma cells (21). However, the roles of DEC1 and DEC2 in apoptosis induced by anti-tumor drugs are still unknown.

In the present study, we examined the effects of DEC1 and DEC2 on the paclitaxel-induced apoptosis of human breast cancer MCF-7 cells. Our results suggest that DEC1 has 
A

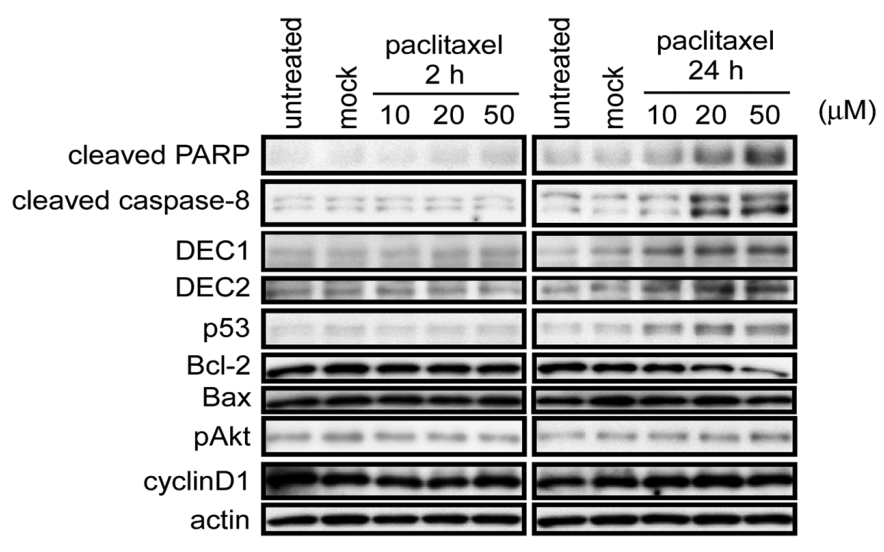

B

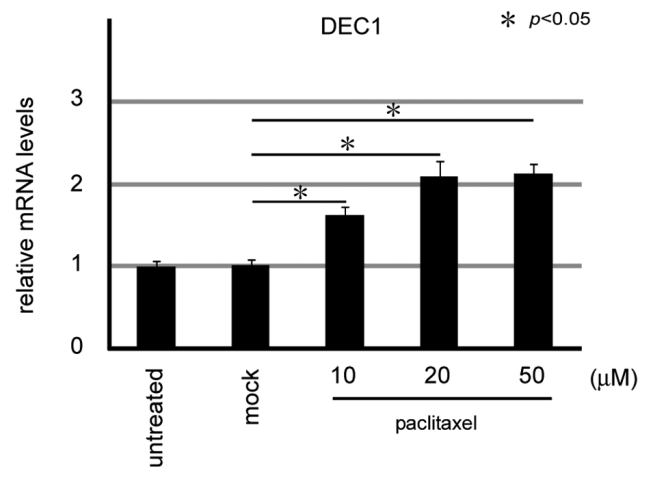

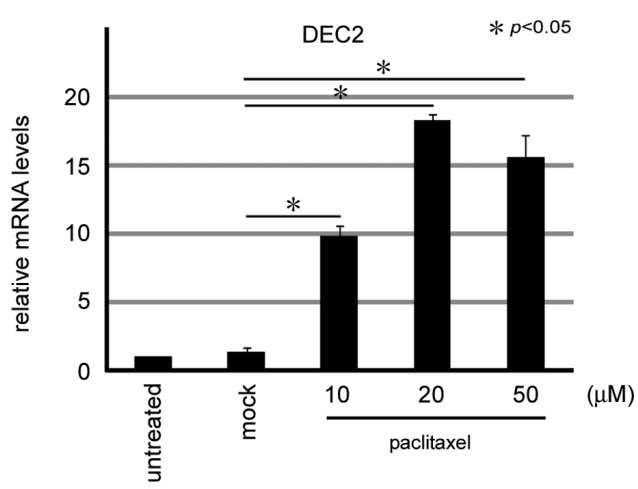

Figure 1. Protein and mRNA levels of DEC1 and DEC2 and apoptosis-related proteins were increased in MCF-7 cells treated with paclitaxel. (A) MCF-7 cells were untreated or treated with the control treatment (buffer alone: Mock) or 10, 20 or $50 \mu \mathrm{M}$ of paclitaxel for 2 or $24 \mathrm{~h}$, and cell lysates were prepared and subjected to Western blot analyses of cleaved PARP, cleaved caspase-8, DEC1, DEC2, p53, Bcl-2, Bax, pAkt, cyclinD1 and actin. One representative of at least three independent experiments with similar results is shown. (B) MCF-7 cells were treated with or without 10, 20 or $50 \mu \mathrm{M}$ of paclitaxel for $24 \mathrm{~h}$, and then subjected to real-time PCR analyses of DEC1 and DEC2. Each value represents the mean + SEM (bars) of three independent experiments ${ }^{*} \mathrm{p}<0.05$, according to the t-test.

pro-apoptotic effects, and DEC2 has anti-apoptotic effects in paclitaxel-treated MCF-7 cells.

\section{Materials and methods}

Cell culture and treatment. The human breast cancer cell line MCF-7 was obtained from the American Type Culture Collection (ATCC, Manassas, VA, USA). The cells were cultured in Dulbecco's modified Eagle's medium-high glucose (Sigma Chemical Co., St. Louis, MO, USA) supplemented with $10 \%$ fetal bovine serum at $37^{\circ} \mathrm{C}$ in a humidified atmosphere of $95 \%$ air and $5 \% \mathrm{CO}_{2}$. In some experiments, the cells were incubated with various concentrations of paclitaxel (Calbiochem, San Diego, CA, USA) for 2 or $24 \mathrm{~h}$.

Real-time polymerase chain reaction $(P C R)$. We prepared three independent RNA samples $(\mathrm{n}=3)$ from the above cells for real-time PCR. Total RNA was isolated, and first-strand cDNA was synthesized as previously described (12). The real-time PCR was performed using SYBR-Green Master mix (Life Technologies, Carlsbad, CA, USA). The sequences and product sizes of the DEC1 and DEC2 primer sets were described in previous reports (12).

Short interference RNA (siRNA). siRNA against DEC1 and DEC2 were synthesized by Qiagen (Hilden, Germany). The sequences of the DEC1, DEC2 and the negative control siRNA were as previously described (12). In the present study we additionally used another siRNA against DEC1 (DEC1 siRNA-2). The sense and anti-sense sequences of DEC1 siRNA-2 were as follows: 5'-GAAGCAUGUGAAAGCACUATT-3' and 5'-UAGUGCUUUCACAUGCUUCAA-3'. For the siRNA transfection experiments, the cells were seeded at $5 \times 10^{4}$ cells per 35-mm well. siRNAs were transfected into the cells $24 \mathrm{~h}$ later using the lipofectamine RNA iMAX reagent (Invitrogen, Carlsbad, CA, USA). After transfection, the cells were incubated for $48 \mathrm{~h}$ and subjected to various analyses.

Western blotting. Cells transfected with siRNA were lysed using M-PER lysis buffer (Thermo Scientific, Rockford, IL, USA), and their protein concentrations were determined using the bicinchoninic acid (BCA) assay. The obtained lysates (10 $\mu \mathrm{g}$ protein) were subjected to SDS-PAGE, and the separated proteins were transferred to PVDF membranes (Immobilon-P, Millipore, Billerica, MA, USA). The ECL, ECL-plus, or ECL-advance Western blotting detection systems (Amersham, Uppsala, Sweden) were used for detection.

Antibodies. The membranes were incubated with antibodies specific to DEC2 (1:40,000; H-72X; Santa Cruz Biotechnology, Inc.), cyclinD1 (1:1,000; Calbiochem), DEC1 (1:10,000; Novus Biologicals, Inc.), p53 (1:2,000; Novus Biologicals, Inc.), 
PARP (1:1,000; Cell Signaling Technology, Inc.), cleaved caspase-8 (1:20,000; Cell Signaling Technology, Inc.), Bax (1:20,000; Santa Cruz Biotechnology), Bcl-2 (1:30,000; Epitomics, Inc., CA, USA), Akt1 phospho (pS473) (1:3,000; Epitomics, Inc.), and actin (1:30,000; Sigma), followed by horseradish peroxidase-conjugated secondary antibody (IBL, Fujioka, Gunma, Japan). The Can Get Signal immunoreaction enhancer solution (Toyobo, Osaka, Japan) or the Immunoshot immunoreaction enhancer solution (Cosmobio, Co., Ltd., Tokyo, Japan) was used to dilute the primary antibody.

Immunofluorescence staining. MCF-7 cells were seeded in a 4-chamber slide glass and fixed as previously described (12). The permeabilized cells were then washed in phosphatebuffered saline (PBS) twice and treated with 5\% normal horse serum in PBS for 30 min (to minimize the non-specific adsorption of antibodies), before being incubated with antimDEC2 (1:300) (12) or anti-mDEC1 (1:200) (22) antibodies at $4^{\circ} \mathrm{C}$ overnight. The cells were then incubated for $1 \mathrm{~h}$ with goat-anti-rabbit IgG antibody conjugated to the Alexa 488 dye (Life Technologies), while nuclear staining was evaluated using 4',6-diamidino-2-phenylindole (DAPI), or Hoechst 33258. Finally, the cells were visualized using confocal laser scanning microscopy (Leica LSM 710, Solms, Germany).

\section{Results}

Paclitaxel up-regulates the expression of DEC1 and DEC2. Since paclitaxel induces apoptosis in breast cancer cells, we investigated whether it induces DEC1 or DEC2 expression in MCF-7 cells. Treatment with paclitaxel for $2 \mathrm{~h}$ had little effect on the protein levels of DEC1, DEC2, cleaved PARP or cleaved caspase-8 (Fig. 1A). However, their levels were significantly increased after $24 \mathrm{~h}$ paclitaxel treatment. The expression of p53 was slightly increased in the cells treated with 10,20 or $50 \mu \mathrm{M}$ of paclitaxel for $24 \mathrm{~h}$, whereas the expression of Bcl-2 was decreased after $50 \mu \mathrm{M}$ of paclitaxel treatment. The expression levels of Bax, pAkt and cyclinD1 were not affected by paclitaxel treatment. In addition to their proteins, the expression levels of DEC1 and DEC2 mRNA were induced by $24 \mathrm{~h}$ paclitaxel treatment (Fig. 1B). The expression of DEC1 was highest after $50 \mu \mathrm{M}$ paclitaxel treatment, and the expression of DEC2 was highest after $20 \mu \mathrm{M}$ paclitaxel treatment. Therefore, we treated the cells with 20 or $50 \mu \mathrm{M}$ of paclitaxel for $24 \mathrm{~h}$ in the subsequent experiments with DEC1 and DEC2, respectively.

Knockdown of DEC1 and DEC2 in the presence of paclitaxel treatment has opposite effects on the apoptosis of MCF-7 cells. DEC1 or DEC2 knockdown by siRNA significantly reduced the expression of DEC1 or DEC2, respectively, with or without paclitaxel treatment (Fig. 2). The amounts of cleaved PARP, cleaved caspase- 8 and $p 53$ were increased after treatment with paclitaxel, and the increased levels were decreased by DEC1 siRNA transfection. On the other hand, the expression of $\mathrm{Bcl}-2$, which was down-regulated by paclitaxel treatment, was up-regulated by DEC1 knockdown. In contrast, DEC2 knockdown increased the amounts of cleaved PARP and caspase-8, even in the absence of paclitaxel (Fig. 2B), as described previously (12). In addition, we found that the expression of p53 was

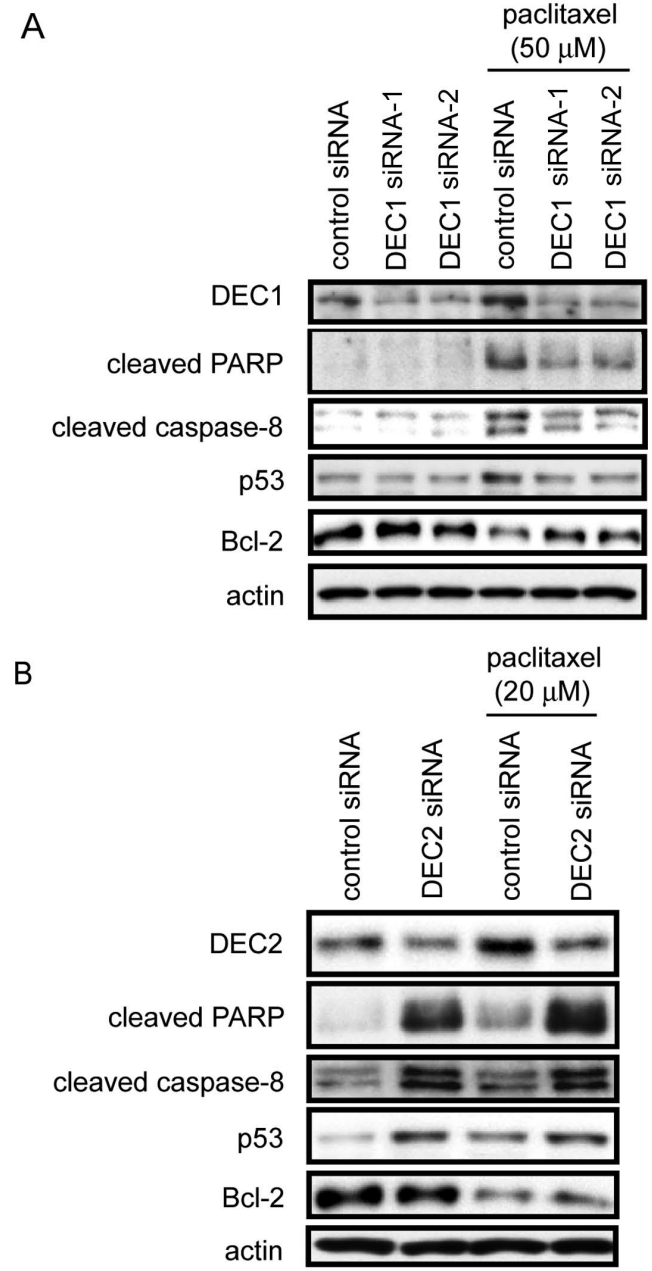

Figure 2. Analyses of apoptosis-associated protein expression in DEC knockdown cells. MCF-7 cells were transfected with control siRNA or siRNA against DEC1 (DEC1 siRNA-1 or DEC1 siRNA-2) or DEC2 and incubated for $48 \mathrm{~h}$, and then the cells were treated with or without paclitaxel (20 or $50 \mu \mathrm{M}$ ) and incubated for $24 \mathrm{~h}$. Then, cell lysates were prepared from the cells and subjected to Western blot analyses of DEC1, DEC2, cleaved PARP, cleaved caspase-8, p53, Bcl-2 and actin, (A) DEC1 siRNA, (B) DEC2 siRNA). One representative of at least three independent experiments with similar results is shown.

up-regulated by DEC2 knockdown in the absence of paclitaxel. DEC2 knockdown also increased the amounts of cleaved PARP and caspase-8, and up-regulated the expression of p53 in the presence of paclitaxel. The expression of $\mathrm{Bcl}-2$ was not affected in the cells treated with DEC2 siRNA, independently of paclitaxel treatment.

Effects of paclitaxel on the subcellular localization of DECI and DEC2. To examine the localization of DEC1 and DEC2 after paclitaxel treatment, MCF-7 cells were stained with antiDEC1 or anti-DEC2 antibodies. As shown in Fig. 3, paclitaxel treatment increased the amount of DEC1 in the nucleus, whereas it increased the amounts of DEC2 in the nucleus and cytoplasm.

Nuclear condensation was increased by DEC2 siRNA treatment, but decreased by DECI siRNA treatment in the presence of paclitaxel. Nuclear condensation is one of the characteristics of apoptosis, which was observed in the MCF-7 cells treated 
A

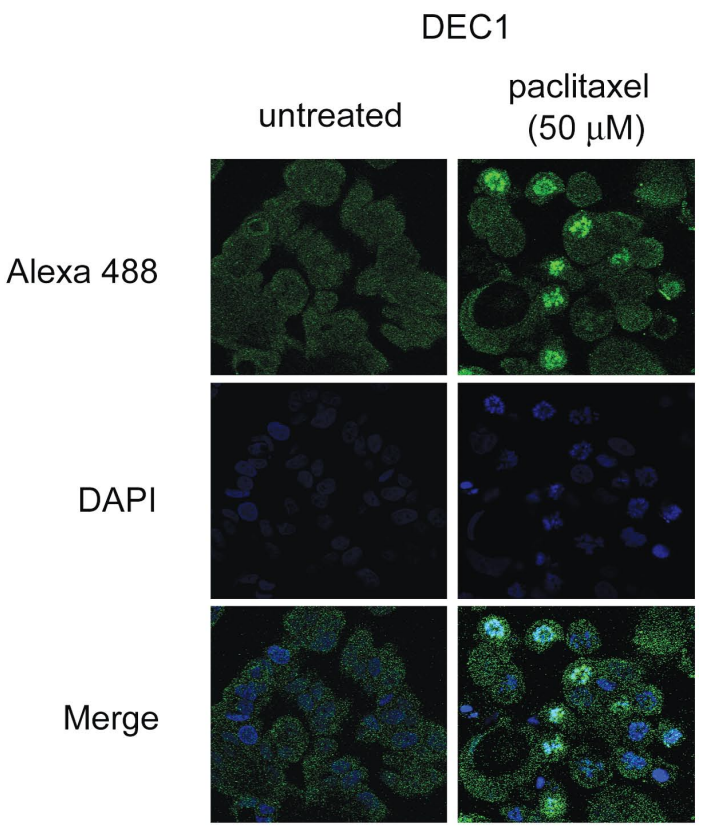

B

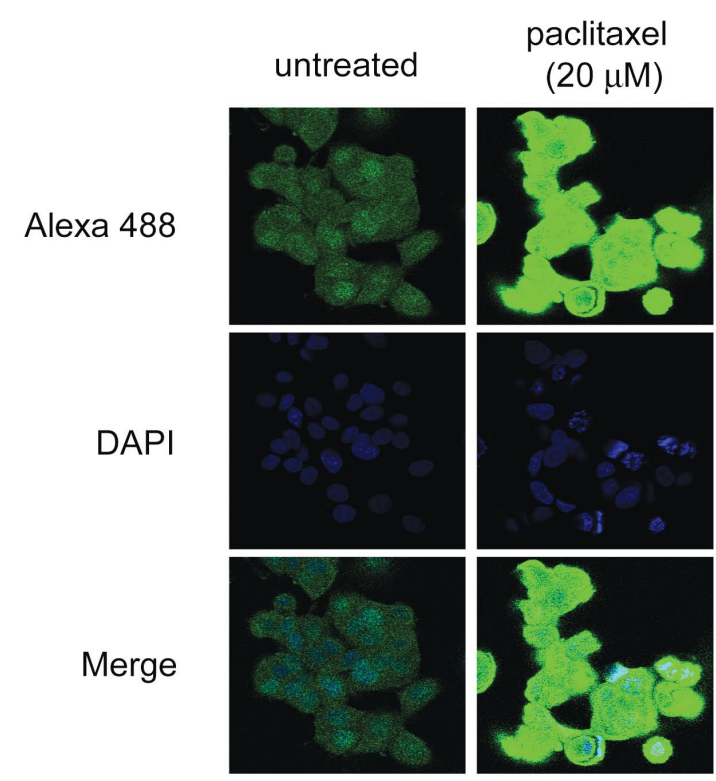

Figure 3. Subcellular localization of DEC1 and DEC2 after paclitaxel treatment. (A and B) MCF-7 cells were treated with or without 20 or $50 \mu \mathrm{M}$ of paclitaxel for $24 \mathrm{~h}$, fixed, incubated with anti-DEC1 or DEC2 antibody, and visualized using Alexa 488-conjugated secondary antibody (green). The cells were also counterstained with DAPI (blue) to detect nuclear staining. An image that is representative of at least two independent experiments with similar results is shown.

with paclitaxel. Knockdown of DEC1 inhibited the nuclear condensation in these cells (Fig. 4A). In contrast, DEC2 knockdown induced nuclear condensation in the absence of paclitaxel and accelerated paclitaxel-induced nuclear condensation (Fig. 4B). These results are consistent with the data from Western blotting shown in Fig. 2.

\section{Discussion}

In the present study, we sought to examine the functions of DEC1 and DEC2 in paclitaxel-induced apoptosis. We found
A

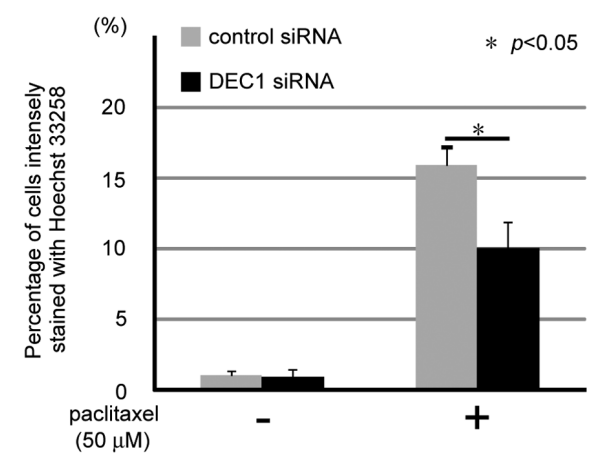

B

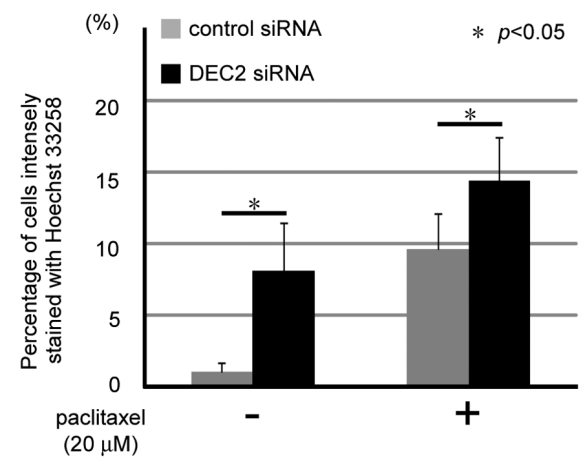

Figure 4. Opposing effects of DEC1 and DEC2 knockdown on apoptosis. (A and B) MCF-7 cells were transfected with control siRNA or siRNA against (A) DEC1 or (B) DEC2, and incubated for $48 \mathrm{~h}$. The cells were then treated with or without paclitaxel $(20$ or $50 \mu \mathrm{M})$ for $24 \mathrm{~h}$. The cells were then stained with Hoechst 33258 . The percentage of cells with intensely fragmented and condensed nuclei was calculated using Hoechst 33258 staining. About 100 cells were examined in ten random microscopic fields at $x 40$ magnification, and each value represents the mean + SEM (bars) of two independent experiments. ${ }^{*} \mathrm{p}<0.05$, according to the t-test.

that paclitaxel increased the expression of DEC1 and DEC2 mRNA and proteins related to apoptosis when it induced the apoptosis in MCF-7 cells, suggesting that paclitaxel treatment up-regulates the transcription of DEC1 and DEC2. There are many environmental and endogenous factors that affect DEC1 expression, such as ionizing-radiation, hypoxia, hormones, growth factors, and cytokines $(11,12,20,23-25)$. On the other hand, the upstream regulators of DEC2 are poorly understood. Here, we found that paclitaxel, an anti-tumor drug, may be one of the upstream factors regulating DEC1 and DEC2 expression.

Our previous reports have demonstrated that DEC1 has pro-apoptotic effects on MCF-7 cells, whereas DEC2 has anti-apoptotic effects, regardless of the presence or absence of TNF- $\alpha$ (12). In this study, we found that DEC1 and DEC2 have opposite effects on apoptosis in MCF-7 cells after paclitaxel treatment, which is not surprising considering that DEC1 and DEC2 mutually regulate each other's expression $(12,26)$. A balance between DEC1 and DEC2 may contribute to the regulation of apoptosis in tumor cells. Immunofluorescent staining demonstrated differences in the subcellular localization of DEC1 and DEC2 after paclitaxel treatment, suggesting differing roles of DEC1 and DEC2 in apoptosis.

Recently, it has been reported that DEC1 is one of the downstream factors of 553 (27). DEC1 expression was induced by the p53 family and by DNA damage in a p53-dependent manner. In the present study, paclitaxel treatment and DEC2 
knockdown increased the expression of p53, whereas DEC1 knockdown down-regulated the p53 expression induced by paclitaxel. In contrast to our observations, a previous study showed that DEC1 overexpression did not affect the expression of p53 in DNA damage-induced premature senescence (27). Thus, the effects of DEC1 on the p53 level differ between paclitaxel induced-apoptosis and DNA damage-induced premature senescence. We speculate that DEC1 and DEC2 are downstream factors of p53 and regulate the expression of p53 through positive or negative feedback mechanisms after exposure to apoptotic insults. We also found that DEC1, but not DEC2, knockdown in the presence of paclitaxel treatment increased the expression of $\mathrm{Bcl}-2$, which is located in the mitochondria and is one of the downstream mediators of p53 $(28,29)$. These findings suggest that, after paclitaxel treatment, DEC1 affects the expression of p53, leading to the activation of mitochondrial apoptotic pathways. In conclusion, we have shown for the first time that the expression levels of DEC1 and DEC2 are up-regulated by paclitaxel treatment. DEC1 and DEC2 may play important roles in the regulation of apoptosis in paclitaxel-treated cells.

\section{Acknowledgements}

This study was supported by Grants-in-Aid for science from the Ministry of Education, Culture, Sports, Science and Technology of Japan and a grant for Hirosaki University Institutional Research.

\section{References}

1. Horwitz SB: Mechanism of action of taxol. Trends Pharmacol Sci 13: 134-136, 1992.

2. Sackett D and Fojo T: Taxanes. Cancer Chemother Biol Response Modif 17: 59-79, 1997.

3. Tudor G, Aguilera A, Halverson DO, Laing ND and Sausville EA Susceptibility to drug-induced apoptosis correlates with differential modulation of $\mathrm{Bad}, \mathrm{Bcl}-2$ and $\mathrm{Bcl}-\mathrm{xL}$ protein levels. Cell Death Differ 7: 574-586, 2000

4. Sunters A, Madureira PA, Pomeranz KM, Aubert M, Brosens JJ, Cook SJ, Burgering BM, Coombes RC and Lam EW: Paclitaxelinduced nuclear translocation of FOXO3a in breast cancer cells is mediated by c-Jun NH2-terminal kinase and Akt. Cancer Res 66: 212-220, 2006

5. Lim SJ, Choi MK, Kim MJ and Kim JK: Alpha-tocopheryl succinate potentiates the paclitaxel-induced apoptosis through enforced caspase 8 activation in human H460 lung cancer cells. Exp Mol Med 41: 737-745, 2009.

6. Kolomeichuk SN, Terrano DT, Lyle CS, Sabapathy K and Chambers TC: Distinct signaling pathways of microtubule inhibitors-vinblastine and Taxol induce JNK-dependent cell death but through AP-1-dependent and AP-1-independent mechanisms, respectively. FEBS J 275: 1889-1899, 2008.

7. Blagosklonny MV, Schulte TW, Nguyen P, Mimnaugh EG, Trepel $J$ and Neckers L: Taxol induction of p21WAF1 and p53 requires c-raf-1. Cancer Res 55: 4623-4626, 1995.

8. Lanni JS, Lowe SW, Licitra EJ, Liu JO and Jacks T: p53-independent apoptosis induced by paclitaxel through an indirect mechanism. Proc Natl Acad Sci USA 94: 9679-9683, 1997.

9. Woods CM, Zhu J, McQueney PA, Bollag D and Lazarides E: Taxol-induced mitotic block triggers rapid onset of a p53-independent apoptotic pathway. Mol Med 1: 506-526, 1995.

10. Saunders DE, Lawrence WD, Christensen C, Wappler NL, Ruan H and Deppe G: Paclitaxel-induced apoptosis in MCF-7 breast-cancer cells. Int J Cancer 70: 214-220, 1997.

11. Sato F, Bhawal UK, Kawamoto T, Fujimoto K, Imaizumi T, Imanaka T, Kondo J,Koyanagi S, Noshiro M, Yoshida $\mathrm{H}$, Kusumi T, Kato Y and Kijima H: Basic-helix-loop-helix (bHLH) transcription factor DEC2 negatively regulates vascular endothelial growth factor expression. Genes Cells 13: 131-144, 2008.
12. Liu Y, Sato F, Kawamoto T, Fujimoto K, Morohashi S, Akasaka H, Kondo J, Wu Y, Noshiro M, Kato Y and Kijima H: Anti-apoptotic effect of the basic helix-loop-helix (bHLH) transcription factor DEC2 in human breast cancer cells. Genes Cells 15: 315-325, 2010.

13. Kondo J, Sato F, Fujimoto K, Kusumi T, Imanaka T, Kawamoto T, Uk B, Noshiro M, Kato Y, Sato T and Kijima H: 57Arg in the bHLH transcription factor DEC2 is essential for the suppression of CLOCK/BMAL2-mediated transactivation. Int J Mol Med 17: 1053-1056, 2006.

14. Chakrabarti J, Turley H, Campo L, Han C, Harris AL, Gatter KC and Fox SB: The transcription factor DEC1 (stra13, SHARP2) is associated with the hypoxic response and high tumour grade in human breast cancers. Br J Cancer 31: 954-958, 2004.

15. Li Y, Zhang H, Xie M, Hu M, Ge S, Yang D, Wan Y and Yan B: Abundant expression of Dec1/stra13/sharp2 in colon carcinoma: its antagonizing role in serum deprivation-induced apoptosis and selective inhibition of procaspase activation. Biochem J 367: 413-422, 2002.

16. Li Y, Xie M, Yang J, Yang D, Deng R, Wan Y and Yan B: The expression of anti-apoptotic protein survivin is transcriptionally upregulated by DEC1 primarily through multiple spl binding sites in the proximal promoter. Oncogene 23: 3298-3306, 2006.

17. Sato F, Kawamoto T, Fujimoto K, Noshiro M, Honda KK, Honma S, Honma K and Kato Y: Functional analysis of the basic helix-loop-helix transcription factor $\mathrm{DEC1}$ in circadian regulation. Interaction with BMAL1. Eur J Biochem 22: 4409-4419, 2004.

18. Nakashima A, Kawamoto T, Honda KK, Ueshima T, Noshiro M, Iwata T, Fujimoto K, Kubo H, Honma S, Yorioka N, Kohno N and Kato Y: DEC1 modulates the circadian phase of clock gene expression. Mol Cell Biol 28: 4080-4092, 2008.

19. Yang XO, Angkasekwinai P, Zhu J, Peng J, Liu Z, Nurieva R, Liu X, Chung Y, Chang SH, Sun B and Dong C: Requirement for the basic helix-loop-helix transcription factor Dec2 in initial TH2 lineage commitment. Nat Immunol 12: 1260-1266, 2009.

20. Thin TH, Li L, Chung TK, Sun H and Taneja R: Stra13 is induced by genotoxic stress and regulates ionizing-radiationinduced apoptosis. EMBO Rep 4: 401-407, 2007.

21. Ehata S, Hanyu A, Hayashi M, Aburatani H, Kato Y, Fujime M, Saitoh M, Miyazawa K, Imamura T and Miyazono K: Transforming growth factor-beta promotes survival of mammary carcinoma cells through induction of antiapoptotic transcription factor DEC1. Cancer Res 67: 9694-9703, 2007.

22. Kawamoto T, Noshiro M, Sato F, Maemura K, Takeda N, Nagai R, Iwata T, Fujimoto K, Furukawa M, Miyazaki K, Honma S, Honma K and Kato Y: A novel autofeedback loop of Decl transcription involved in circadian rhythm regulation. Biochem Biophys Res Commun 313: 117-124, 2004.

23. Miyazaki K, Kawamoto T, Tanimoto K, Nishiyama M, Honda H and Kato Y: Identification of functional hypoxia response elements in the promoter region of the DEC1 and DEC2 genes. J Biol Chem 277: 47014-47021, 2002.

24. Yamada K, Kawata H, Shou Z, Mizutani T, Noguchi T and Miyamoto K: Insulin induces the expression of the SHARP-2/ Stra13/DEC1 gene via a phosphoinositide 3-kinase pathway. J Biol Chem 278: 30719-30724, 2003.

25. Kon N, Hirota T, Kawamoto T, Kato Y, Tsubota T and Fukada Y: Activation of TGF-beta/activin signalling resets the circadian clock through rapid induction of Decl transcripts. Nat Cell Biol 10: 1463-1469, 2008.

26. Azmi S, Sun H, Ozog A and Taneja R: mSharp-1/DEC2, a basic helix-loop-helix protein functions as a transcriptional repressor of E box activity and Stra13 expression. J Biol Chem 278: 20098-20109, 2003

27. Qian Y, Zhang J, Yan B and Chen X: DEC1, a basic helix-loophelix transcription factor and a novel target gene of the p53 family, mediates p53-dependent premature senescence. J Biol Chem 283: 2896-2905, 2008.

28. Messmer UK, Reed UK and Brüne B: Bcl-2 protects macrophages from nitric oxide-induced apoptosis. J Biol Chem 271: 20192-20197, 1996.

29. Yin XM: Signal transduction mediated by Bid, a pro-death Bcl-2 family protein, connects the death receptor and mitochondria apoptosis pathways. Cell Res 10: 161-167, 2000. 